\title{
Prevalencia de hipotiroidismo y su asociación con factores de riesgo cardiometabólicos en mujeres adultas argentinas
}

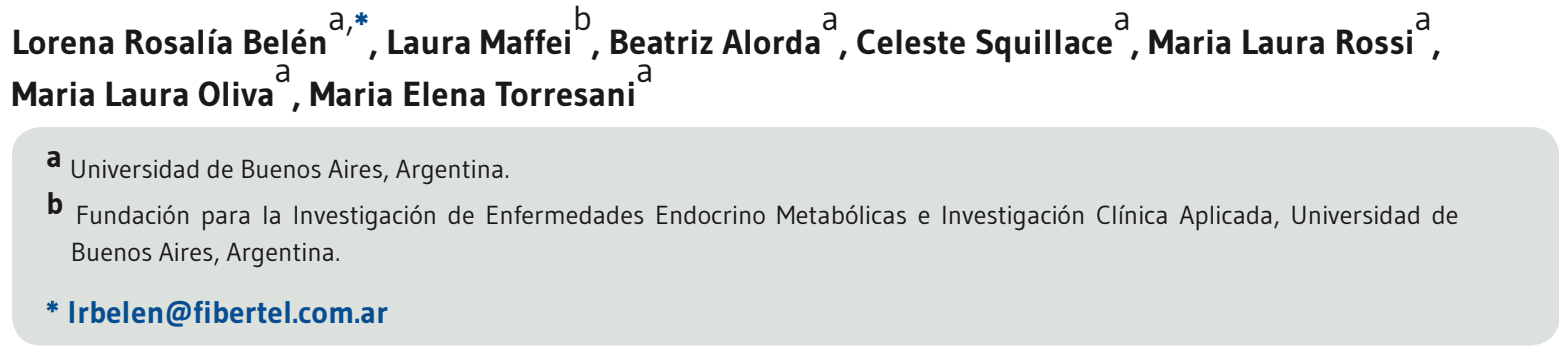

Recibido el 9 de Julio de 2015; aceptado el 20 de agosto de 2015.

\section{PALABRAS CLAVE \\ Evaluación tiroidea; \\ Menopausia; \\ Estado nutricional; \\ Factores de riesgo cardiometabólicos.} Prevalencia de hipotiroidismo y su asociación con factores de riesgo cardiometabólicos en mujeres
adultas argentinas

\section{RESUMEN}

Introducción: El hipotiroidismo se asocia con factores de riesgo cardiometabólicos (FRC) además de la edad y del sexo femenino. El principal objetivo fue conocer la prevalencia de hipotiroidismo y su asociación con FRC en mujeres adultas concurrentes a un Centro de Endocrinología de la Ciudad Autónoma de Buenos Aires, Argentina.

Material y Métodos: Diseño transversal y observacional en muestra no probabilística de 316 mujeres adultas (40 a 65 años), categorizadas en hipotiroideas controladas con tratamiento farmacológico y eutiroideas. Variables: Se estudiaron los siguientes FRC: Índice Masa Corpo$\mathrm{ral}$ (IMC), $<25 \mathrm{~kg} / \mathrm{m}^{2}$ y $\geq 25,0 \mathrm{Kg} / \mathrm{m}^{2}$; circunferencia cintura (CC) $\leq 88 \mathrm{~cm}$ y $>88 \mathrm{~cm}$; etapa biológica (premenopausia y posmenopausia), tabaquismo, sedentarismo; perfil lipídico alterado (ColT>200mg/dl; LDL-c>130mg/dl; Tg>150mg/dl; HDL-c<40mg/dl); HTA $\geq 120 / 80 \mathrm{mmHg}$ y glucemia basal aumentada $>100 \mathrm{mg} / \mathrm{dl}$. Estadística con SPSS 15.0 estableciendo medidas de tendencia central, ANOVA y OR con intervalos de confianza 95\% (IC) y diferencia de proporciones con valor $p<0,05$.

Resultados: 46,2\% hipotiroideas controladas y 53,8\% eutiroideas; $64,2 \%$ posmenopáusicas; $68 \%$ con sobrepeso u obesidad y $45,2 \%$ con CC aumentada. La edad fue similar en ambos grupos: 53,3 (DS=6,4) años hipotiroideas y 53,9 (DS=6,6) años eutiroideas. Sedentarismo fue el FRC más prevalente en ambos grupos. Las hipotiroideas presentaron más riesgo de IMC aumentado $(O R=0,36 \mathrm{IC} 95 \%=0,21-0,59 ; p=0,0001)$ y $C C$ alterada $(O R=0,53 \mathrm{IC} 95 \%=0,34-0,84$; $\mathrm{p}=0,006)$ que las eutiroideas.

Conclusiones: La prevalencia de hipotiroidismo se presentó aproximadamente en la mitad de la muestra. El estado nutricional alterado fue el único FRC asociado significativamente con el hipotiroidismo. 


\section{KEYWORDS}

Thyroid evaluation;

Menopause;

Nutritional

assessment;

Cardiometabolic risk factors.
Prevalence in hypothyroid and its association with cardiometabolic risk factor argentine adult women

\section{ABSTRACT}

Introduction: It is known that hypothyroidism is associated with cardiometabolic risk factors (CRF) as well as age, female gender. The main objective was determine the prevalence of hypothyroidism and its association with cardiometabolic risk factors in adult women attending the Foundation for Endocrine Metabolic Diseases Research of the Autonomous City of Buenos Aires, Argentina.

Material and Methods: Cross-sectional and observational design. Probabilistic sample of 316 adult women (40-65 years), randomized into two groups: hypothyroid controlled with pharmacological treatment and euthyroid. Variable: the following were studied CRF: body mass index (BMI), normal $<25 \mathrm{~kg} / \mathrm{m}^{2}$ and increased $\geq 25.0 \mathrm{~kg} / \mathrm{m}^{2}$; waist circumference (WC) normal $\leq 88 \mathrm{~cm}$ and increased $>88 \mathrm{~cm}$; biological stage (premenopausal and postmenopausal), smoking, sedentary lifestyle; altered lipid profile (TC>200mg/dl; LDL $>130 \mathrm{mg} / \mathrm{dl} ; \mathrm{Tg}>150 \mathrm{mg} / \mathrm{dl}$; HDL-c $<40 \mathrm{mg} / \mathrm{dl}$ ); HT $\geq 120 / 80 \mathrm{mmHg}$ and increased fasting glucose $>100 \mathrm{mg} / \mathrm{dl}$. Statistics with SPSS 15.0 establishing measures of central tendency, ANOVA and OR with 95\% confidence intervals $(\mathrm{Cl})$ and difference of proportions with $\mathrm{p}<0.05$.

Results: The $46.2 \%$ of the sample were women controlled hypothyroid and euthyroid $53.8 \%$. The $64.2 \%$ were postmenopausal, $68 \%$ overweight or obese and $45.2 \%$ with WC increased. Age was similar in both groups: $53.3(S D=6.4)$ years the hypothyroid and $53.9(S D=6.6)$ years the euthyroid. The inactivity was the CFR more prevalent in both groups. The hypothyroid women had a significantly higher risk for $\mathrm{BMI}$ increased $(\mathrm{OR}=0.36 ; 95 \% \mathrm{Cl}=0.21-0.59 ; \mathrm{p}=0.0001)$ and WC altered $(\mathrm{OR}=0.53 ; 95 \% \mathrm{Cl}=0.34-0.84 ; \mathrm{p}=0.006)$ with respect to the euthyroid women.

Conclusions: Found a prevalence of hypothyroidism in approximately half of the sample. The altered nutritional status CRF was the only significantly associated with hypothyroidism.

CITA

Belén LR, Maffei L, Alorda B, Squillace C, Rossi ML, Oliva ML, Torressani ME. Prevalencia de hipotiroidismo y su asociación con factores de riesgo cardiometabólicos en mujeres adultas argentinas. Rev Esp Nutr Hum Diet. 2015; 19(3): 146 - 152. DOI: 10.14306/renhyd.19.3.157

\section{INTRODUCCIÓN}

Dentro de las disfunciones tiroideas el hipotiroidismo es altamente frecuente a nivel mundial, siendo de 4 a 5 veces más prevalente en las mujeres y especialmente a medida que se incrementa la edad ${ }^{1,2}$. Se han encontrado cifras del 8 al 10\% en mujeres a partir de los 40 años, afectando a más del $12 \%$ de las mujeres mayores de 60 años; algunos estudios señalan incluso que puede alcanzar cifras de hasta el $30 \%{ }^{3,4}$.

Este aumento de prevalencia en las mujeres fue relacionado con mayor susceptibilidad a alteraciones de la autoinmunidad, variaciones fisiológicas y hormonales del sistema endocrino en diferentes etapas de la vida ${ }^{5,6,7}$.

Si bien se desconoce el porqué de esta mayor susceptibilidad, se encontró asociación con las concentraciones de estrógenos y los cambios hormonales, siendo en consecuencia la perimenopausia un período de mayor propensión ${ }^{8}$.

Los signos y síntomas del hipotiroidismo suelen ser difíciles de diferenciar clínicamente de los presentes en el síndrome del climaterio y el envejecimiento, por lo que se puede dificultar el diagnóstico de la enfermedad ${ }^{9,10}$.

El hipotiroidismo se asocia con factores de riesgo cardiometabólicos como hipertensión arterial (HTA), dislipemia, inflamación sistémica y resistencia a la insulina (RI), siendo considerada esta disfunción tiroidea factor independiente para el desarrollo de enfermedad cardiovascular ${ }^{11,12,13}$. 
A su vez, en diferentes investigaciones se han reportado tanto en situaciones de hipotiroidismo ${ }^{14,15}$ como en la perimenopausia ${ }^{16,17,18}$ ganancia de peso y modificaciones en la composición corporal a expensas de un aumento de la masa grasa.

El objetivo de este estudio fue conocer la prevalencia de hipotiroidismo y su asociación con factores de riesgo cardiometabólicos en una muestra de mujeres adultas concurrentes a control nutricional.

\section{MATERIAL Y MÉTODOS}

Se llevó a cabo un diseño transversal y observacional sobre una muestra voluntaria por conveniencia de mujeres de 40 a 65 años no embarazadas ni con menopausia precoz (antes de los 40 años), que concurrieron por primera vez a la consulta nutricional de la Fundación para la Investigación de las Enfermedades Endocrino Metabólicas (FIEEM) e Investigación Clínica Aplicada (ICA) en la Ciudad Autónoma de Buenos Aires. Fueron categorizadas según el diagnóstico de la evaluación tiroidea en hipotiroideas controladas con tratamiento farmacológico y mujeres aparentemente sanas, sin diagnóstico de hipotiroidismo en su historia clínica, excluyéndose aquellas mujeres donde no constaba este dato.

Se estudiaron las siguientes variables consideradas factores de riesgo cardiometabólicos: estado nutricional, medido a través del Índice de Masa Corporal (IMC), normal $<25 \mathrm{~kg} / \mathrm{m}^{2}$ o aumentado $\geq 25 \mathrm{~kg} / \mathrm{m}^{2}$; circunferencia de la cintura (CC), normal $\leq 88 \mathrm{~cm}$ o aumentada $>88 \mathrm{~cm}$; perfil lipídico alterado (ColT>200mg/dl; LDL>130mg/dl; $\mathrm{Tg}>150 \mathrm{mg} / \mathrm{dl} ; \mathrm{HDL}<40 \mathrm{mg} / \mathrm{dl}$ ); HTA $\geq 120 / 80 \mathrm{mmHg}$; glucemia basal aumentada $>100 \mathrm{mg} / \mathrm{dl}$; etapa biológica (pre y posmenopausia), hábito tabáquico y sedentarismo.

\section{Recolección de los datos y análisis estadístico}

Se realizó medición directa de peso, talla y CC según técnicas estandarizadas. El peso se registró en kilogramos $(\mathrm{kg})$, pesando a la paciente en balanza tipo CAM, con precisión de $0,1 \mathrm{~kg}$ con el mínimo de ropa. La talla se midió en metros (m), por medio de un tallímetro con una precisión de 0,01m, con la paciente de pie en posición firme con la cabeza mantenida según el plano de Frankfort. Se relacionó el peso y la talla a través del IMC y se consideró sobrepeso u obesidad a valores iguales o superiores a $25,0 \mathrm{~kg} / \mathrm{m}^{219}$. Se midió la CC a la altura recomendada por la OMS, en el punto medio entre el reborde costal (la duodécima costilla) y la cresta ilíaca.

Los datos sobre la etapa biológica y actividad física se obtuvieron por interrogación directa. Se clasificó en premenopausia desde los 40 años de edad hasta el momento de la menopausia. Y posmenopausia al período posterior a la menopausia, hasta los 65 años de edad. La división de estas dos etapas fue dada por la menopausia, considerando a este momento según la Organización Mundial de la Salud (OMS), como el cese definitivo de los ciclos menstruales determinado retrospectivamente luego de 12 meses consecutivos de amenorrea que no responda a otra causa fisiológica o patológica ${ }^{20}$.

Se consideró sedentarismo a la falta de actividad física programada por lo menos 3 veces por semana 50 a 60 minutos cada vez, necesaria para alcanzar las recomendaciones mundiales dadas por la OMS de un mínimo de 150 minutos semanales de ejercicio aeróbico moderado ${ }^{21}$.

Los datos del perfil lipídico fueron obtenidos directamente de la historia clínica correspondientes a un tiempo retrospectivo no mayor a 6 meses.

Para el análisis estadístico de los datos se empleó el paquete estadístico SPSS 15.0, estableciendo medidas de tendencia central, ANOVA para comparación de medias, OR con intervalos de confianza (IC) al 95\% de seguridad y $x^{2}$ para diferencia de proporciones. En todos los casos se trabajó con nivel de significación <0,05.

\section{Consideraciones éticas}

Con el fin de salvaguardar los derechos, la seguridad y el bienestar de las pacientes encuestadas, se sometió el proyecto a la evaluación del Comité de Revisión Institucional (CRI) para su aprobación correspondiente antes de iniciar el reclutamiento de la información.

\section{RESULTADOS}

La muestra quedó conformada por 316 mujeres (en la Tabla 1 se presenta la caracterización) de las cuales 146 (46,2\%) fueron hipotiroideas controladas con tratamiento farmacológico y 170 (53,8\%) eutiroideas. El 64,2\% se presentó en etapa posmenopáusica, el $68 \%$ con sobrepeso u obesidad según el IMC y el $45,2 \%$ con CC aumentada.

En el grupo de mujeres hipotiroideas la edad promedio fue de $53,3(\mathrm{DS}=6,4)$ años, el IMC de $28,9(\mathrm{DS}=4,7) \mathrm{kg} / \mathrm{m}^{2}$ y la CC de $90,4(D S=10,8) \mathrm{cm}$. En el grupo de mujeres eutiroideas los valores promedios respectivos fueron 53,9 (DS=6,6) años, $27,8(\mathrm{DS}=4,8) \mathrm{kg} / \mathrm{m}^{2}$ y $87,9(\mathrm{DS}=10,3) \mathrm{cm}$. Por comparación de medias se observaron valores ligeramente superiores pero 
Tabla 1. Caracterización de la muestra de mujeres adultas.

Fundación para la Investigación de las Enfermedades Endocrino Metabólicas.

\begin{tabular}{|c|c|c|c|c|c|c|c|}
\hline \multicolumn{2}{|c|}{ Caracterización de la muestra } & \multicolumn{2}{|c|}{$\begin{array}{l}\text { Hipotiroideas } \\
\text { (n: 146) }\end{array}$} & \multicolumn{2}{|c|}{$\begin{array}{l}\text { Eutiroideas } \\
\text { (n: 170) }\end{array}$} & \multicolumn{2}{|c|}{$\begin{array}{l}\text { Totales } \\
\text { (n: 316) }\end{array}$} \\
\hline \multirow{3}{*}{ Etapa Biológica } & & $\mathrm{n}$ & $\%$ & $\mathbf{n}$ & $\%$ & $\mathbf{n}$ & $\%$ \\
\hline & Premenopáusicas & 55 & 17,4 & 58 & 18,4 & 113 & 35,8 \\
\hline & Posmenopáusicas & 91 & 28,8 & 112 & 35,4 & 203 & 64,2 \\
\hline \multirow[t]{4}{*}{ Estado Nutricional } & IMC $<25 \mathrm{~kg} / \mathrm{m}^{2}$ & 30 & 9,5 & 71 & 22,5 & 101 & 32,0 \\
\hline & $I M C \geq 25 \mathrm{~kg} / \mathrm{m}^{2}$ & 116 & 36,7 & 99 & 31,3 & 215 & 68,0 \\
\hline & $\mathrm{CC} \leq 88 \mathrm{~cm}$ & 68 & 21,5 & 105 & 33,3 & 173 & 54,8 \\
\hline & $\mathrm{CC}>88 \mathrm{~cm}$ & 78 & 24,6 & 65 & 20,6 & 143 & 45,2 \\
\hline
\end{tabular}

Gráfico 1. Evaluación tiroidea y estado nutricional en mujeres adultas.

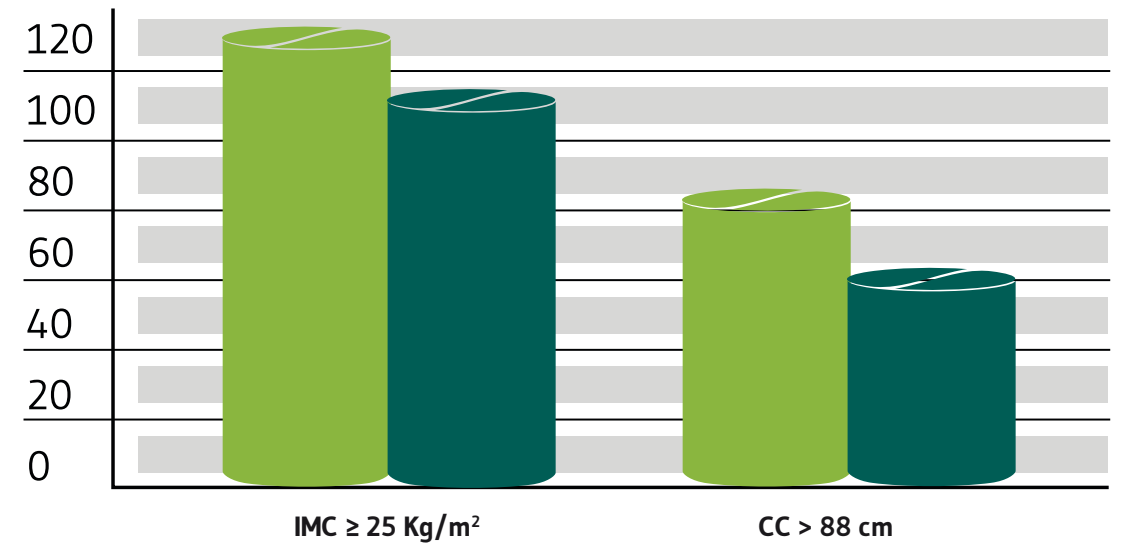

Hipotiroideas

Eutiroideas significativos del IMC $(p=0,04)$ y de la CC $(p=0,03)$ en el grupo de hipotiroideas respecto de las eutiroideas (Tabla 2).

Según se muestra en la Tabla 3, el sedentarismo fue el factor de riesgo cardiometabólico más prevalente en ambos grupos de mujeres estudiadas $(84,9 \%$ en las hipotiroideas y $80,6 \%$ en las eutiroideas).
Las mujeres hipotiroideas presentaron 0,36 veces más riesgo (IC95\%:0,21-0,59) de sobrepeso u obesidad (p:0,0001) y 0,53 veces más riesgo (IC95\%:0,34-0,84) de tener la CC aumentada $(\mathrm{p}: 0,006)$ respecto a las mujeres eutiroideas. Los demás factores de riesgo cardiometabólicos estudiados no se asociaron con el estado tiroideo (Tabla 3). 


\section{DISCUSIóN}

La mayoría de los estudios reportan que la incidencia tanto de hipotiroidismo franco como subclínico, se incrementa a medida que el individuo se aproxima a la quinta década de vida. Se registra mayor prevalencia en mujeres, especialmente en las edades comprendidas entre 65 y 79 años de $\operatorname{edad}^{22,23,24}$.

La pandemia de la obesidad a nivel mundial ha impulsado un nuevo interés en la relación entre las hormonas tiroideas y el peso corporal25. La disfunción tiroidea presenta una relación bien establecida con el peso corporal, registrándose frecuentemente correlación entre los niveles de TSH y el IMC ${ }^{26}$.

Aunque es bien sabido que las hormonas tiroideas juegan un papel clave en la regulación de la homeostasis de la energía ${ }^{27}$, aún no existen fuertes evidencias sobre la identificación de los mecanismos por los que la obesidad puede alterar el eje hipotalámico pituitario-tiroideo (HPT). Una de las hipótesis que explican el efecto directo de la TSH está dada porque su receptor se expresa en el tejido adiposo ${ }^{28}$.

Tabla 2. Valoración antropométrica de la muestra - Valores medios y desvíos.

Fundación para la Investigación de las Enfermedades Endocrino Metabólicas.

\begin{tabular}{l|c|c|c|c|c} 
Valoración medios & $\begin{array}{c}\text { Hipotiroideas } \\
\text { (n: 146) }\end{array}$ & \multicolumn{2}{|c|}{$\begin{array}{c}\text { Eutiroideas } \\
\text { (n: 170) }\end{array}$} & $\begin{array}{c}\text { Diferencia } \\
\text { de medias }\end{array}$ \\
\cline { 1 - 4 } & $\mathrm{x}$ & $\mathrm{DE}$ & $\mathrm{x}$ & $\mathrm{DE}$ & $\mathrm{p}$ \\
\hline Edad (años) & 53,3 & 6,4 & 53,9 & 6,6 & 0,41 \\
IMC (kg/m²) & 28,9 & 4,7 & 27,8 & 4,8 & $\mathbf{0 , 0 4}$ \\
CC (cm) & 90,4 & 10,8 & 87,9 & 10,3 & $\mathbf{0 , 0 3}$
\end{tabular}

x: media

DE: desvío estándar

p: significativa $<0,05$

Tabla 3. Prevalencia de factores de riesgo cardiometabólicos en mujeres adultas según el estado tiroideo. Fundación para la Investigación de las Enfermedades Endocrino Metabólicas

\begin{tabular}{|c|c|c|c|c|c|c|c|c|}
\hline \multirow[t]{2}{*}{$\begin{array}{l}\text { Factores de Riesgo } \\
\text { Cardiometabólicos }\end{array}$} & \multicolumn{2}{|c|}{$\begin{array}{l}\text { Hipotiroideas } \\
\text { (n: 146) }\end{array}$} & \multicolumn{2}{|c|}{$\begin{array}{l}\text { Eutiroideas } \\
\text { (n: 170) }\end{array}$} & \multirow{3}{*}{$\begin{array}{l}\text { OR } \\
0,36\end{array}$} & \multirow{3}{*}{$\begin{array}{c}\text { IC95\% } \\
\text { 0,21-0,59 }\end{array}$} & \multirow{3}{*}{$\begin{array}{c}X^{2} \\
16,25\end{array}$} & \multirow{3}{*}{$\begin{array}{c}P \\
0,0001\end{array}$} \\
\hline & $\mathrm{n}$ & $\%$ & $\mathrm{n}$ & $\%$ & & & & \\
\hline$I M C \geq 25,0 \mathrm{~kg} / \mathrm{m}^{2}$ & 116 & 79,4 & 99 & 58,2 & & & & \\
\hline $\mathrm{CC}>88 \mathrm{~cm}$ & 78 & 53,4 & 65 & 38,2 & 0,53 & $0,34-0,84$ & 7,31 & 0,006 \\
\hline Posmenopausia & 91 & 62,3 & 112 & 65,9 & 1,16 & $0,73-1,85$ & 0,43 & 0,51 \\
\hline Hábito tabáquico & 16 & 10,9 & 20 & 11,8 & 1,08 & $0,53-2,17$ & 0,05 & 0,82 \\
\hline Sedentarismo & 124 & 84,9 & 137 & 80,6 & 0,73 & $0,40-1,33$ & 1,03 & 0,31 \\
\hline $\mathrm{Col} \mathrm{T}>200 \mathrm{mg} / \mathrm{dl}$ & 108 & 73,9 & 112 & 65,9 & 0,67 & $0,41-1,10$ & 2,43 & 0,11 \\
\hline $\mathrm{LDL}>130 \mathrm{mg} / \mathrm{dl}$ & 76 & 52,0 & 97 & 57,1 & 1,22 & $0,78-1,90$ & 0,79 & 0,37 \\
\hline $\mathrm{HDL}<40 \mathrm{mg} / \mathrm{dl}$ & 5 & 3,4 & 6 & 3,5 & 1,03 & $0,30-3,45$ & 0,002 & 0,95 \\
\hline $\mathrm{TG}>150 \mathrm{mg} / \mathrm{dl}$ & 39 & 26,7 & 44 & 25,9 & 0,95 & $0,57-1,58$ & 0,02 & 0,86 \\
\hline $\mathrm{TA} \geq 120 / 80 \mathrm{mmHg}$ & 58 & 39,7 & 56 & 32,9 & 0,74 & $0,47-1,18$ & 1,57 & 0,21 \\
\hline $\mathrm{GL}>100 \mathrm{mg} / \mathrm{dl}$ & 48 & 32,9 & 42 & 24,7 & 0,66 & $0,41-1,09$ & 2,57 & 0,10 \\
\hline
\end{tabular}


Son varios los grupos de investigación que han informado asociación entre el aumento de las concentraciones de TSH y valores elevados de circunferencia de la cintura y el IMC 29,30 .

En una cohorte de 201 mujeres italianas entre 18 y 68 años de edad, el aumento de los niveles de TSH se asoció con el IMC y la circunferencia de la cintura, independientemente de otros parámetros metabólicos. Los autores postulan a la progresiva acumulación de grasa central posiblemente como un fenómeno termogénico de adaptación ${ }^{31}$.

Sin embargo, en trabajos como el de Westerink y Col. se vio que el aumento de los niveles plasmáticos de TSH dentro del rango normal, se asocia con adiposidad visceral en los pacientes con enfermedad vascular manifiesta por encima de la edad de 66 años, no encontrándose asociación entre los niveles plasmáticos de TSH y el peso corporal o el IMC ${ }^{32}$.

En nuestra investigación las mujeres hipotiroideas presentaron significativamente valores mayores de IMC y de CC respecto de las mujeres eutiroideas. A su vez, fue mayor la prevalencia de sobrepeso $u$ obesidad y de CC alterada en el grupo de mujeres hipotiroideas.

En nuestra muestra no fue analizado el valor de TSH. Si bien todas las mujeres hipotiroideas estaban controladas con tratamiento hormonal sustitutivo, podría esto representar una limitación en el trabajo a la hora de poder comparar con otros estudios. Por otro lado, tampoco se controló en la muestra el uso de terapia de reemplazo hormonal (TRH). Se ha informado que en pacientes menopáusicas con hipotiroidismo que reciben terapia hormonal sustitutiva, se generan cambios en la concentración de tiroxina libre y $\mathrm{TSH}^{33}$.

A pesar de que recientes estudios concluyen que el hipotiroidismo se asocia con aterosclerosis y enfermedad arterial coronaria11,12,13, en nuestro trabajo no se observó asociación con los demás factores de riesgo cardiovascular estudiados.

Por último, si bien en general la disfunción tiroidea es más prevalente en individuos obesos, se necesitan más estudios para evaluar la relación causa-efecto entre la obesidad y el hipotiroidismo ${ }^{34}$. Dado que existen investigaciones que sugieren modificaciones en el peso corporal con modestos incrementos de valores de TSH dentro de los rangos de referencia ${ }^{25}$, se sugiere continuar la línea de investigación incluyendo mayor número de pacientes e incorporar en el análisis los valores de las hormonas tiroideas. Identificar cambios tempranos en la función tiroidea, como un factor de riesgo para el aumento de peso, podría ayudar a la prevención y tratamiento oportuno del sobrepeso u obesidad.

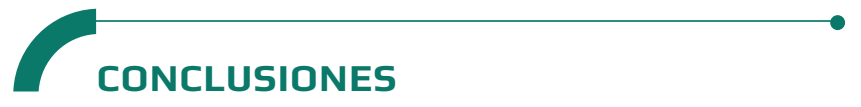

Aproximadamente la mitad de las mujeres estudiadas presentan hipotiroidismo, controlado con tratamiento farmacológico.

No se observó diferencias de edad entre las mujeres hipotiroideas y las eutiroideas, ni aumento de prevalencia de hipotiroidismo en la posmenopausia.

Independientemente del estado tiroideo, el sedentarismo fue el factor de riesgo cardiometabólico más prevalente.

En las mujeres hipotiroideas fue significativamente mayor el riesgo de sobrepeso u obesidad y circunferencia de cintura alterada, así como los valores medios hallados de ambos parámetros antropométricos.

No hubo asociación entre los demás factores de riesgo cardiometabólicos estudiados y el estado tiroideo.

\section{CONFLICTO DE INTERESES}

Los autores manifiestan no presentar ningún conflicto de interés que haya afectado el informe final del presente trabajo.

\section{BIBLIOGRAFÍA}

1. Habra M, Sarlis NJ. Thyroid and aging. Rev Endocr Metab Disord. 2005; 6(2): 145-54.

2. Hunter I, Greene SA, MacDonald TM, Morris AD. Prevalence and aetiology of hypothyroidism in the young. Arch Dis Chile. 2000; 83(3): 207-10.

3. Zárate A, Saucedo $R$, Basurto $L$, Hernández-Valencia $M$. Principales problemas de salud en la mujer adulta. Acta Med Gpo Ang. 2006; 4: 57-60.

4. Sender Palacio MJ, Vernet Vernet M, Pérez Lopez S, Faro Colomés M, Rojas Blanc M, Pallisa Gabril L. Enfermedad funcional tiroidea en la población de edad avanzada. Aten Primaria. 2004; 34(4): 192-7.

5. Gaches F, Delaire L, Nadalon S, Loustaud-Ratt V, Vidal E. Frequency of autoimmune diseases in 218 patients with autoimmune thyroid pathologies. Rev Med Interne. 1998; 19: 173-9.

6. Weetman AP. Autoimmune thyroiditis: predisposition and pathogenesis. Clin Endocrinol (Oxf). 1992; 36(4): 307-23.

7. Zárate A, Basurto L, Saucedo R y Hernandez Valencia M. Enfermedad tiroidea: un tema de revisión constante por el 
ginecólogo, por su frecuencia en las mujeres. Ginecol Obstet Mex. 2009; 77: 96-102.

8. Hernandez Valencia M, Córdova Pérez N, Zárate A, Basurto L, Manuel Apolinar L, Ruiz My Col. Modificación de la agudización de los síntomas de la menopausia e hipotiroidismo. Ginec Obstet Mex. 2008; 76(10): 571-5.

9. Schindler AE. Thyroid function and postmenopause. Gynecol Endocrinol. 2003; 17(1): 79-85.

10. Zárate A, Basurto L, Hernández-Valencia M. Los trastornos tiroideos en la mujer. Ginecol Obstet Mex. 2001; 69: 200-5.

11. Völzke H, Schwahn C, Wallaschofski H, Dörr M. Review: The association of thyroid dysfunction with all-cause and circulatory mortality: is there a causal relationship? J Clin Endocrinol Metab. 2007; 92(7): 2421-9.

12. Razvi S, Shakoor A, Vanderpump M, Weaver JU, Pearce SH. The influence of age on the relationship between subclinical hypothyroidism and ischemic heart disease: a metaanalysis. ] Clin Endocrinol Metab. 2008; 93(8): 2998-3007.

13. Kim SK, Kim SH, Park KS, Park SW, Cho YW. Regression of the increased common carotid artery-intima media thickness in subclinical hypothyroidism after thyroid hormone replacement. Endocr J. 2009; 56(6): 753-8.

14. Wolf M, Weigert A, Kreymann G: Body composition and energy expenditure in thyroidectomized patients during shortterm hypothyroidism and thyrotropinsuppressive thyroxine therapy. Eur J Endocrinol. 1996; 134: 168-73.

15. Seppel T, Kosel A, Schlaghecke R. Bioelectrical impedance assessment of body composition in thyroid disease. Eur ] Endocrinol. 1997; 136(5): 493-8.

16. Torresani ME, Oliva ML, Echevarría C, Rossi ML, Maffei L. Perfil Antropométrico de Mujeres Perimenopáusicas. Rev Esp Nutr Comunitaria. 2007; 13(1): 6-10.

17. Pavon de Paz C, Alameda H y Roldan JO. Obesidad y Menopausia. Nutr Hosp. 2006; 21(6): 633-7.

18. Torresani ME, Oliva ML, Echevarría C, Rossi ML, Maffei L. Factores predictores de la ganancia de peso corporal a partir de la menopausia. En prensa.

19. (US) NOEIEP on the I Evaluation, and Treatment of Obesity in Adults. Clinical Guidelines on the Identification, Evaluation, and Treatment of Overweight and Obesity in Adults. National Heart, Lung, and Blood Institute; 1998.

20. World Health Organization. Report of a WHO Scientific Group: Research on the Menopause in the 1990's. Geneva, Switzerland: World Health Organization. WHO Technical report Series 866; 1996.

21. World Health Organization (WHO). The Health Benefits of
Physical Activity in Developing Countries; Geneva: WHO; 2005

22. Weetman AP. Hypothyroidism: screening and subclinical disease. BM]. 1997; 314(7088): 1175-8.

23. Karlin NJ, Weintraub N, Chopra IJ. Current controversies in endocrinology: screening of asymptomatic elderly for subclinical hypothyroidism. J Am Med Dir Assoc. 2004; 5(5): 333-6

24. Biondi B, Cooper DS. The clinical significance of subclinical thyroid dysfunction. Endocr Rev. 2008; 29(1): 76-131.

25. Reinehr T. Obesity and thyroid function. Mol Cell Endocrinol. 2010; 316(2): 165-71.

26. lacobellis G, Ribaudo MC, Zappaterreno A, lannucci CV, Leonetti F. Relationship of thyroid function with body mass index, leptin, insulin sensitivity and adiponectin in euthyroid obese women. Clin Endocrinol (Oxf). 2005; 62(4): 487-91.

27. Yen PM. Physiological and molecular basis of thyroid hormone action. Physiol Rev. 2001; 81(3): 1097-142.

28. Peeters RP, van der Deure WM, van den Beld AW, van Toor $H_{\text {, }}$ Lamberts SW, Janssen JA, et al. The Asp727Glu polymorphism in the TSH receptor is associated with insulin resistance in healthy elderly men. Clin Endocrinol (Oxf). 2007; 66(6): 80815.

29. Fox CS, Pencina MJ, D'Agostino RB, Murabito JM, Seely EW, Pearce EN, et al. Relations of thyroid function to body weight: cross-sectional and longitudinal observations in a communitybased sample. Arch Intern Med. 2008; 168(6): 587-92.

30. Waterhouse DF, McLaughlin AM, Walsh CD, Sheehan F, O'Shea D. An examination of the relationship between normal range thyrotropin and cardiovascular risk parameters: a study in healthy women. Thyroid. 2007; 17(3): 243-8.

31. De Pergola G, Ciampolillo A, Paolotti S, Trerotoli P, Giorgino R. Free triiodothyronine and thyroid stimulating hormone are directly associated with waist circumference, independently of insulin resistance, metabolic parameters and blood pressure in overweight and obese women. Clin Endocrinol (Oxf). 2007; 67(2): 265-9.

32. Westerink J, van der Graaf Y, Faber DR, Visseren FL; SMART study group. The relation between thyroid-stimulating hormone and measures of adiposity in patients with manifest vascular disease. Eur ] Clin Invest. 2011; 41(2): 159-66.

33. Pines A, Dotan I, Tabori U, Villa Y, Mijatovic V, Levo Y, Ayalon D. L-thyroxine prevents the bone-conserving effect of HRT in postmenopausal women with subclinical hypothyroidism. Gynecol Endocrinol. 1999; 13(3): 196-201.

34. Verma A, Jayaraman M, Kumar HK, Modi KD. Hypothyroidism and obesity. Cause or effect? Saudi Med J. 2008; 29(8): 1135-8. 